\title{
BMJ Open Study protocol: NITric oxide during cardiopulmonary bypass to improve Recovery in Infants with Congenital heart defects (NITRIC trial): a randomised controlled trial
}

\begin{abstract}
Luregn J Schlapbach, ${ }^{\oplus 1,2}$ Stephen Brian Horton, ${ }^{\circledR 3,4,5}$ Debbie Amanda Long, ${ }^{1,2}$ John Beca, ${ }^{6}$ Simon Erickson, ${ }^{7}$ Marino Festa, ${ }^{8,9}$ Yves d'Udekem, ${ }^{10,11,12,13}$ Nelson Alphonso, ${ }^{14}$ David Winlaw, ${ }^{15,16}$ Kerry Johnson, ${ }^{1,2}$ Carmel Delzoppo, ${ }^{5,17}$ Kim van Loon, ${ }^{18}$ B Gannon, ${ }^{19}$ Jonas Fooken, ${ }^{19}$ Antje Blumenthal, ${ }^{20}$ Paul Young, ${ }^{21}$ Mark Jones, ${ }^{22}$ Warwick Butt, ${ }^{5,17}$ Andreas Schibler, ${ }^{1,2}$ On behalf of the NITRIC Study Group, the Australian and New Zealand Intensive Care Society Clinical Trials Group (ANZICS CTG), the Paediatric Critical Care Research group (PCCRG) and the ANZICS Paediatric Study Group (PSG)
\end{abstract}

To cite: Schlapbach LJ, Horton SB, Long DA, et al. Study protocol: NITric oxide during cardiopulmonary bypass to improve Recovery in Infants with Congenital heart defects (NITRIC trial): a randomised controlled trial. BMJ Open 2019;9:e026664. doi:10.1136/ bmjopen-2018-026664

- Prepublication history for this paper is available online To view these files, please visit the journal online (http://dx.doi. org/10.1136/bmjopen-2018026664).

Received 19 November 2018 Revised 15 May 2019 Accepted 20 June 2019

Check for updates

(c) Author(s) (or their employer(s)) 2019. Re-use permitted under CC BY-NC. No commercial re-use. See rights and permissions. Published by BMJ.

For numbered affiliations see end of article.

Correspondence to Dr Andreas Schibler; a.schibler@uq.edu.au

\section{ABSTRACT}

Introduction Congenital heart disease (CHD) is a major cause of infant mortality. Many infants with CHD require corrective surgery with most operations requiring cardiopulmonary bypass (CPB). CPB triggers a systemic inflammatory response which is associated with low cardiac output syndrome (LCOS), postoperative morbidity and mortality. Delivery of nitric oxide (NO) into CPB circuits can provide myocardial protection and reduce bypassinduced inflammation, leading to less LCOS and improved recovery. We hypothesised that using NO during CPB increases ventilator-free days (VFD) (the number of days patients spend alive and free from invasive mechanical ventilation up until day 28) compared with standard care. Here, we describe the NITRIC trial protocol.

Methods and analysis The NITRIC trial is a randomised, double-blind, controlled, parallel-group, two-sided superiority trial to be conducted in six paediatric cardiac surgical centres. One thousand three-hundred and twenty infants $<2$ years of age undergoing cardiac surgery with CPB will be randomly assigned to NO at $20 \mathrm{ppm}$ administered into the CPB oxygenator for the duration of CPB or standard care (no N0) in a 1:1 ratio with stratification by age ( $<6$ and $\geq 6$ weeks), single ventricle physiology $(\mathrm{Y} / \mathrm{N})$ and study centre. The primary outcome will be VFD to day 28. Secondary outcomes include a composite of LCOS, need for extracorporeal membrane oxygenation or death within 28 days of surgery; length of stay in intensive care and in hospital; and, healthcare costs. Analyses will be conducted on an intention-to-treat basis. Preplanned secondary analyses will investigate the impact of NO on host inflammatory profiles postsurgery. Ethics and dissemination The study has ethical approval (HREC/17/QRCH/43, dated 26 April 2017), is registered in the Australian New Zealand Clinical Trials Registry (ACTRN12617000821392) and commenced recruitment in

\section{Strengths and limitations of this study}

- This study tests the efficacy and safety of a simple intervention during cardiopulmonary bypass to improve early postoperative morbidity.

- The intervention aims to reduce patient-centred adverse outcomes after a common high-risk procedure for the most common congenital condition.

- The study includes follow-up of neurodevelopmental outcome and quality of life which will allow assessment of the long-term impact of the intervention.

- The study includes biobanking to investigate the biological mechanisms underlying the clinical findings in anxillary studies.

- The study will be the largest randomised controlled trial performed in paediatric cardiac surgery to date.

July 2017. The primary manuscript will be submitted for publication in a peer-reviewed journal.

Trial registration number ACTRN12617000821392

\section{INTRODUCTION}

Congenital heart disease (CHD) is the most common congenital condition, affecting around one in a hundred live born children. ${ }^{1}$ Up to $50 \%$ need cardiac surgery to correct the underlying abnormality at some stage during their life, with the majority of procedures requiring cardiopulmonary bypass (CPB). Substantial reductions in perioperative mortality in children following cardiac surgery have been achieved, ${ }^{2}$ and adult survivors of CHD now outnumber paediatric patients 
with CHD in most high-income countries. ${ }^{34}$ Despite these advances, major postoperative morbidity remains common and is associated with increased rate of long-term mortality, morbidity and disability. ${ }^{5}$ The exposure of host blood to large artificial organ surfaces combined with myocardial injury during surgery, results in a strong systemic inflammatory response of the host, which is further aggravated by reperfusion injury and the release of damage-associated molecular patterns during surgery. ${ }^{6}$ Endotoxin release, leucocyte and complement activation, widespread activation of inflammatory mediators and endothelial leak ${ }^{7}$ postoperatively contribute to low cardiac output syndrome (LCOS) ${ }^{89}$ Postoperative LCOS is clinically defined by a need for inotropes to maintain end organ perfusion, an increased arterial-venous oxygen extraction, lactataemia and oliguria. LCOS may lead to multiorgan failure and a need for extracorporeal life support (ECLS) ${ }^{10}$ Several studies have shown that the presence and severity of LCOS, which affects $25 \%-40 \%$ of children post-CPB in the first hours following heart surgery, ${ }^{11}$ is strongly associated with postoperative morbidity and mortality. CPB-related side effects are most pronounced in infants and young children ${ }^{12}$ due to their higher metabolic requirement, altered inflammatory response and higher CPB circuit to patient blood volume. At the same time, this age group is exposed to CPB during a vulnerable phase of brain development ${ }^{13-15}$ and remains at highest risk of suffering neurological impairments ${ }^{16}$ due to acute brain injury occurring within the context of LCOS. Recent trials to reduce LCOS during cardiac surgery for CHD testing interventions such as steroids ${ }^{17}$ have not demonstrated consistent benefit. ${ }^{18}$ Given the adverse effects of CPB on early recovery and longterm neurodevelopment, there remains an urgent need for clinical trials evaluating novel therapies to address these problems. ${ }^{5}$

Nitric oxide (NO) is an endogenous anti-inflammatory mediator ${ }^{19}$ and is essential to regulate endothelial function and microvascular inflammation. ${ }^{20}$ Several studies have demonstrated that exogenous NO can reduce myocardial damage in clinical and experimental settings of ischaemia and reperfusion. ${ }^{21-24} \mathrm{~A}$ previous small single-centre study in 16 children reported a reduction in bypass-induced inflammation using gaseous $\mathrm{NO}$ delivered at $20 \mathrm{ppm}$ to CPB circuits. ${ }^{25}$ The duration of mechanical ventilation was significantly shorter ( 8.4 vs 16.3 hours; $\mathrm{p}<0.05$ ) and so was intensive care unit (ICU) length of stay ( 53.8 vs 79.4 hours; $\mathrm{p}<0.05$ ) in children receiving $\mathrm{NO}$ compared with the placebo group. We have previously reported the feasibility and safety of NO delivery to CPB in a single-centre randomised controlled pilot study in 198 infants and children (0-16 years) undergoing cardiac surgery. ${ }^{26}$ This pilot study demonstrated a statistically significantly lower proportion of children with LCOS in the intervention arm, a reduced requirement for ECLS and a trend to reduced length of stay, and shorter duration of mechanical ventilation. The effect was greatest in children under 2 years of age, with the greatest treatment benefit observed in children under 6 weeks.

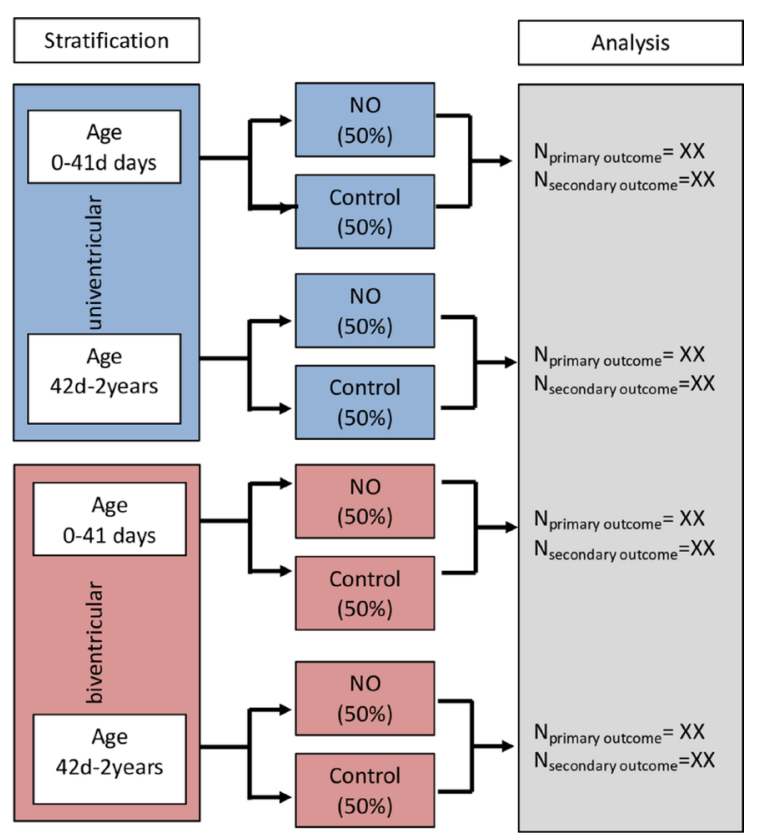

Figure 1 Study flow diagram. NO, nitric oxide.

Accordingly, we designed the NITRIC trial to test the primary hypothesis that, in infants under 2 years having cardiac surgery, using NO during CPB increases ventilator-free days (VFD) (the number of days patients spend alive and free from invasive mechanical ventilation up until postoperative day 28) compared with standard care. Here, we describe the NITRIC trial protocol.

\section{METHODS}

The NITRIC trial is a 1320 patient multicentre, randomised, double-blind, standard care-controlled, parallel-group, trial in infants and children $<2$ years of age undergoing open heart surgery on CPB (figure 1).

\section{Study setting}

Tertiary/quaternary paediatric cardiac surgical services in Australia and New Zealand, including Cardiac and Paediatric Intensive Care Services of Royal Children's Hospital, Melbourne; Starship Children's Hospital Auckland NZ; The Children's Hospital at Westmead, Sydney; Princess Margaret Hospital for Children, Perth; and Queensland Children's Hospital, Brisbane and Utrecht Medical Center, Utrecht, The Netherlands.

\section{Participants}

Eligible children will be identified in the preoperative clinics, in the general cardiac wards or in the neonatal or paediatric intensive care unit (PICU). Included will be infants and children $<2$ years of age undergoing elective open heart surgery on CPB where consent of parents/ guardian is obtained prior to surgery. The exclusion criteria relate to patient characteristics that will interfere with consent, with the intervention or with measurement of the primary and secondary outcomes. Inclusion and exclusion criteria are shown in table 1. 
Table 1 Inclusion and exclusion criteria

\begin{tabular}{|c|c|c|}
\hline Patient group & Criterium & Definition \\
\hline \multirow[t]{3}{*}{ Inclusion } & Age & - Postnatal age <2years. \\
\hline & Procedure & Open elective heart surgery. \\
\hline & Consent & - Parental/caregiver consent available prior to surgery. \\
\hline \multirow[t]{9}{*}{ Exclusion } & Age & Age $\geq 2$ years. \\
\hline & & - Heart surgery not requiring cardiopulmonary bypass. \\
\hline & Consent & - Lack of parental/caregiver consent. \\
\hline & Pulmonary hypertension & $\begin{array}{l}\text { Persistently elevated pulmonary vascular resistance preoperatively receiving inhaled } \\
\text { NO or preoperative intravenous use of drugs involved in the NO pathway such as } \\
\text { glyceryl trinitrate within } 48 \text { hours prior to CPB (oral sildenafil treatment alone is not } \\
\text { an exclusion). }\end{array}$ \\
\hline & & $\begin{array}{l}\text { Treated with high doses of vasoactive drugs defined as a Vasoactive-Inotrope } \\
\text { Score } \geq 15 \text { within } 24 \text { hours prior to surgery.* }\end{array}$ \\
\hline & & Cardiac arrest within 1 week (7 days) prior to surgery. \\
\hline & & $\begin{array}{l}\text { Acute respiratory distress syndrome requiring high-frequency oscillatory ventilation } \\
\text { within } 48 \text { hours prior to surgery. }\end{array}$ \\
\hline & & $\begin{array}{l}\text { Chronic ventilator dependency (patients treated with non-invasive or invasive } \\
\text { ventilation continuously for }>28 \text { days prior to cardiopulmonary bypass). }\end{array}$ \\
\hline & & - Pre-existing methaemoglobinemia (>3\%). \\
\hline
\end{tabular}

*Gaies MG, Gurney JG, Yen AH, et al. Vasoactive-inotropic score as a predictor of morbidity and mortality in infants after cardiopulmonary bypass. Pediatr Crit Care Med 2010;11:234-8.

CPB, cardiopulmonary bypass; ECLS, extracorporeal life support; NO, nitric oxide.

Enrolment of patients undergoing repeated surgery during the first 2 years of life

In order to assess the impact of the intervention on longterm outcomes, patients who were previously enrolled and randomised into the study who require a second or subsequent surgical procedure (such as a patient with single ventricle physiology requiring a staged palliation) will undergo the same treatment allocation for subsequent surgeries requiring $\mathrm{CPB}$, unless parents opt out. Patients who were not recruited into the study during their first procedure, but are scheduled for a subsequent procedure requiring $\mathrm{CPB}$ prior to their second birthday, will be eligible for recruitment.

\section{Randomisation and blinding}

Treatment assignment will be performed using a secure, centralised, web-based randomisation interface (Research Electronic Data Capture (REDCap), ${ }^{27}$ The University of Queensland). The allocation sequence will be generated by the study statistician using computer-generated random numbers using a variable block size stratified by age ( $<6$ weeks, 6 weeks to 24 months), univentricular versus biventricular lesions, and by site. Of the investigating team, only the study perfusionist will be aware of the randomisation and NO delivery. Cardiologists, cardiac surgeons, anesthesiologists, intensivists, PICU nurses, research assistants, data analysts, and parents and caregivers will be blinded for the intervention. Rationale for stratification: the age group under 6 weeks represents the cohort at highest surgical risk. In the pilot study, the effect size of the NO delivery was greatest in these infants. ${ }^{26}$ Cardiac physiology (univentricular vs biventricular) is a major determinant of surgical complexity, risk and outcome. ${ }^{15}$

\section{Blinding of the intervention}

Blinding arrangements in the operating theatre will be achieved by covering the NO delivery system with drapes. The dedicated study NO delivery system will be connected to the CPB oxygenator at all times, independent of randomisation. The family, surgeons, anaesthetists and PICU staff will be not aware of the treatment arm a patient is allocated to. The perfusionists will be advised that all aspects of CPB except for provision of NO (or not) should be performed according to standard institutional practice for study participants.

\section{Interventions}

Infants will be randomly assigned to $\mathrm{NO}$ or standard care. Those allocated to the $\mathrm{NO}$ arm will receive $\mathrm{NO}$ during $\mathrm{CPB}$ 
blended into the fresh gas flow of the CPB oxygenator, which is kept at $3 \mathrm{~L} / \mathrm{min}$. NO levels are maintained at 20 ppm using a NO delivery system (Ikaria INOmax DSIR, Ikaria, NJ, USA) or similar device. Continuous sampling of $\mathrm{NO}$ and $\mathrm{NO}_{2}$ concentration will be undertaken from an access port before the oxygenator. $\mathrm{NO}$ will be started immediately when the patient is placed on CPB and ceased once weaned off CPB. Patients allocated to the standard study arm will receive the standard gase oxygen-air mix into the $\mathrm{CPB}$ oxygenator at a flow rate of $3 \mathrm{~L} / \mathrm{min}$. If patients require several $\mathrm{CPB}$ runs during the same procedure, the study treatment will be provided for each $\mathrm{CPB}$ run using the same treatment allocation for every CPB run.

\section{Relevant concomitant perioperative care}

Arterial partial pressures of $\mathrm{CO}_{2}$ will be maintained constantly in both study arms as per institutional practice (following each centre's protocols on alpha/ $\mathrm{pH}$ stat and temperatures). The $\mathrm{FiO}_{2}$ of the fresh gas flow will be set between $21 \%$ and $100 \%$, according to centre-specific CPB protocols. Techniques of anaesthesia and surgery will not be specified to allow site-specific individual practice. The decision whether a patient requires treatment with inhaled NO (iNO) into the ventilator circuit prior to, during or after CPB remains at the discretion of the treating physicians (anaesthetists, cardiac surgeons or intensivists) independent of treatment allocation. The postoperative care and decisions on inotropes and other vasoactive drug delivery, fluid management, renal replacement therapy, iNO therapy or indication for ECLS will be performed as per site-specific standard protocols of care.

\section{Study outcomes}

The primary outcome is VFD for the first 28 days postrandomisation (table 2). The primary outcome will be measured using duration of invasive respiratory support for all episodes with an endotracheal tube in situ for the first 28 days postrandomisation. A systematic zero value will be assigned for patients who die to weigh death as the most pejorative outcome. Treatment with non-invasive ventilation and high-flow nasal cannulae will not be considered as ventilator days. Secondary outcomes are defined as the composite outcome compromising LCOS, need for postoperative ECLS or 28-day mortality; ICU and hospital length of stay; and healthcare costs. In addition, the long-term outcome of patients will be followed up at 12 months postprocedure.

Low cardiac output syndrome

$\mathrm{LCOS}^{10}$ is defined as a blood lactate level $>4 \mathrm{mmol} / \mathrm{L}$ with an oxygen extraction of $>35 \% \quad\left(\mathrm{SaO}_{2}-\mathrm{ScvO}_{2}\right.$ gradient $>35 \%$ ) within the first 48 hours postoperatively, or a high inotrope requirement defined as Vasoactive-Inotrope Score $\geq 15$. 2829

\section{Rationale for primary and secondary outcomes}

VFD represents one of the strongest predictors of shortterm and long-term outcomes, ${ }^{10}$ including length of ICU stay, morbidity (impaired neurodevelopment, hospital-acquired infections) and mortality. VFD directly reflect intensive care resource use and healthcare costs. ${ }^{30}$ VFD fulfils Specific, Measurable, Achievable, Realistic and Timely criteria. LCOS is directly related to VFD, as infants usually do not tolerate weaning or fail extubation if LCOS and organ dysfunction have not resolved. The composite of LCOS, ECLS use and mortality as a secondary end point, is a strong patient-centred outcome and directly relates to the intervention in terms of biological plausibility.

\section{Adverse events}

It is recognised that the postoperative paediatric cardiac surgical patient population will experience a number of common aberrations in laboratory values, signs and symptoms due to the severity of the underlying disease and the impact of standard therapies. Intensive care patients will frequently develop life-threatening organ failure(s) unrelated to study interventions and despite optimal management. Therefore, consistent with established practice in academic ICU trials, ${ }^{31}$ events that are part of the natural history of the primary disease process or expected complications of critical illness will not be reported as serious adverse events in this study. All adverse events which are considered to be potentially causally related to the study intervention or are otherwise of concern in the investigator's judgement will be reported unless they are prespecified study outcomes. Specific adverse events related to NO delivery during CBP include air embolism, severe hypotension during bypass and increased methaemoglobinemia $(>3 \%)$. Of note, in previous studies, methaemoglobin values using $\mathrm{NO}$ at $20 \mathrm{ppm}$ were similar in both the control and intervention groups (1.4\%). ${ }^{25}{ }^{26}$ Events that are collected as study outcomes will not be reported as adverse events.

\section{Safety data monitoring}

The Data Safety Monitoring Board (DSMB) consists of a general and a cardiac paediatric intensivist, a cardiac surgeon and a statistician. None of the DSMB members will be involved in recruitment of study patients at their site. DSMB members will not be supervised by any study investigator or participate as investigators in any study currently under review by this DSMB. The primary objective of the DSMB is to monitor the safety of the intervention and the validity and integrity of the data from the NITRIC study. Additionally, the DSMB will evaluate the pace of recruitment and will make recommendations to the NITRIC Chief investigator(s) and Steering Board regarding the continuation, modification or termination of the study. The DSMB will evaluate on an ongoing basis, the accumulating safety assessments to ensure the ongoing safety of study subjects. The DSMB will meet via teleconference call after recruitment of 660, and of 1000 children, respectively, and on trial completion.

The DSMB can request unblinding in the event of a serious adverse event defined as a cardiac arrest, need for ECLS, or other incident leading to permanent harm considered to be likely related to the study intervention. 


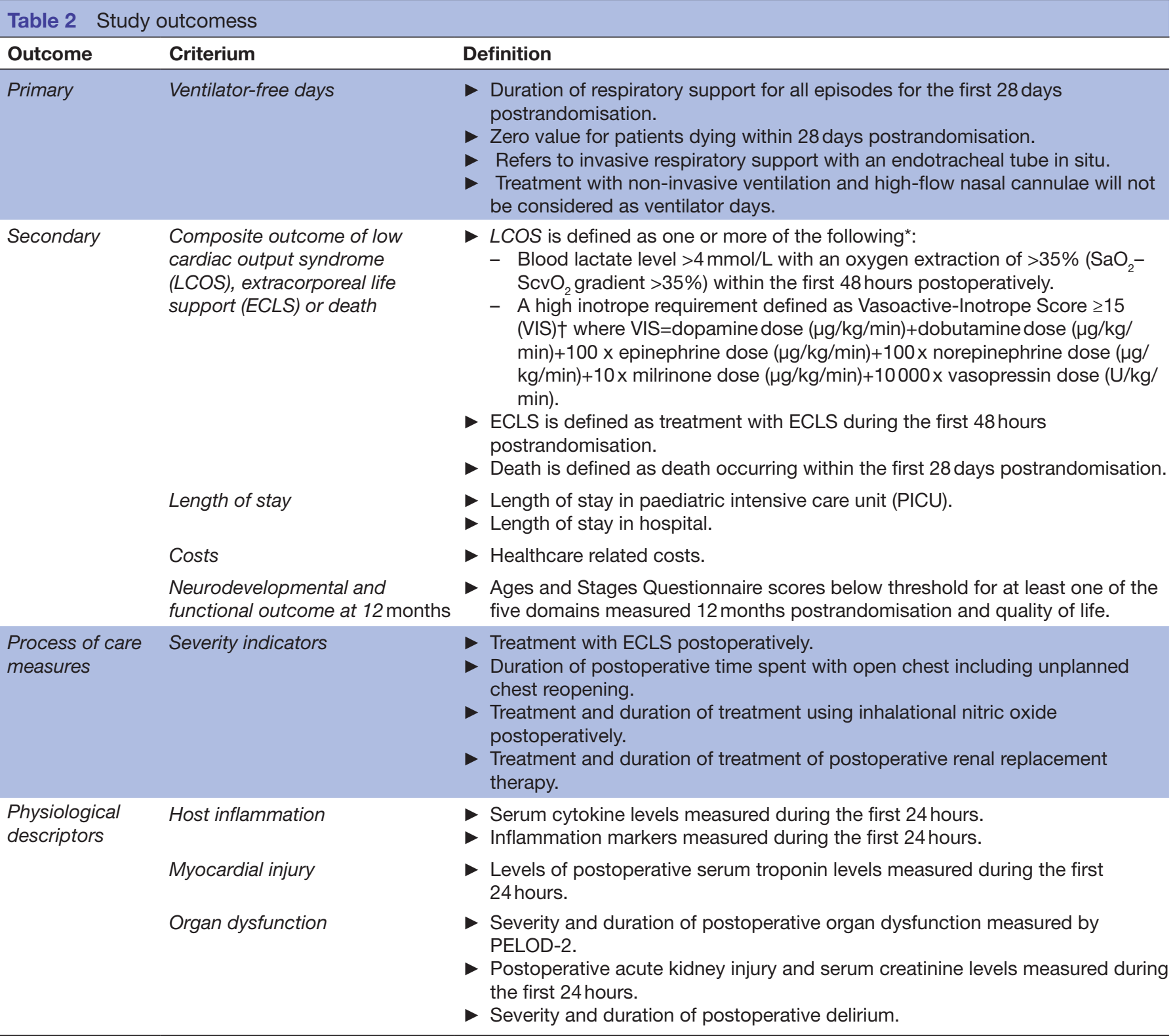

*Hoffman TM, Wernovsky G, Atz AM, et al. Efficacy and safety of milrinone in preventing low cardiac output syndrome in infants and children after corrective surgery for congenital heart disease. Circulation 2003;107:996-1002.

†Gaies MG, Gurney JG, Yen AH, et al. Vasoactive-inotropic score as a predictor of morbidity and mortality in infants after cardiopulmonary bypass. Pediatr Crit Care Med 2010;11:234-8.

PELOD-2, Paediatric Logistic Organ Dysfunction-2.

\section{Sample size}

A pilot study of 134 patients aged $<2$ years showed a 2.74 days (66 hours) increase in VFD associated with the study intervention. ${ }^{26}$ This includes patients who died, who were considered as zero VFDs. The VFD increase associated with the intervention represents an effect size of $0.33 \mathrm{SD}$ based on a SD of 8.1 days in the pilot study control group. Based on the primary outcome measure VFD, 1320 patients (660 per group) will be required to demonstrate a significant increase in VFD assuming a minimally clinically significant effect size $(0.2 \mathrm{SD} ; 1.66$ days or 40 hours), $90 \%$ power, two-sided alpha level of significance of $5 \%, 10 \%$ withdrawals and $15 \%$ increase in sample size to account for a non-normal distribution of VFD. In Australia and New Zealand, $\sim 800$ of children $<2$ years of age undergo surgery for a congenital heart defect requiring $\mathrm{CPB}$ each year, including patients with multiple procedures. The consent rate of eligible patients was $78 \%$ in the pilot trial. ${ }^{26}$ With an expected conservative estimate $60 \%$ enrolment rate of eligible patients, we expect a 3.5-year recruitment period for the study.

\section{Data collection, management and analysis}

Data collection

Baseline variables (demographics, primary cardiac diagnosis, comorbidities including syndromes), preoperative disease 
severity, surgical data (length of CPB and cross-clamp, type of surgery and complexity score, other CPB characteristics, blood product usage), primary end points, secondary end points, predetermined physiological variables of interest and process of care measures will be prospectively recorded into a study REDCap online database. Plausibility and range checks are implemented. Paired arterial and venous gases will be collected postoperatively at $0,6,12,24$ and 48 hours (in PICU until discharge to the ward or removal of arterial and central venous lines, whichever occurs first) to assess for lactate and $\mathrm{SaO}_{2}-\mathrm{ScvO}_{2}$ gradient. Key physiological and blood parameters and Paediatric Logistic Organ Dysfunction-2 scores ${ }^{32}$ will be measured at 0, 6, 12, 24 and 48 hours postadmission or until PICU discharge whichever occurs earlier. Delayed chest closure, use of iNO and duration of circulatory, renal and ventilatory support postoperatively will be recorded. Gross functional performance assessment will be recorded on admission to PICU and on discharge from hospital. ${ }^{33}$ Neurological and functional outcome (including phone interviews with parents/caregivers) will be assessed at 12 months postoperatively using Ages and Stages Questionnaires ${ }^{345}$ and assessment of quality of life using paediatric quality of life inventory. ${ }^{36}$ Details on the long-term follow-up will be published separately.

\section{Biobanking}

Blood markers for myocardial injury and inflammatory response will be collected on induction of anaesthesia (baseline-prebypass), at admission to PICU (0 hourspostbypass) and at 12 , and 24 hours, in patients where parents consent to biobanking. Blood will be collected presurgery as preoperative baseline (before onset of cardiac surgery, done by the anaesthetist during the induction of the patient once the arterial line has been inserted): $1-2 \mathrm{~mL}$ of EDTA blood (for DNA), $2.5 \mathrm{~mL}$ of PAXgene blood (for gene expression markers), $1-2 \mathrm{~mL}$ of serum; and postoperatively at 0,12 and 24 hours (in PICU until discharge to the ward): $2.5 \mathrm{~mL}$ of PAXgene blood (time point 0 ), and $1-2 \mathrm{~mL}$ of serum. The samples will be processed, stored and shipped according to accepted international standards and batch analysed.

The investigators are responsible for ensuring the accuracy, completeness, legibility and timeliness of the data reported. The investigators will maintain adequate case histories of study participants, including accurate case report forms (CRFs), and source documentation. Data will be prospectively entered into a secure web-based database (REDCap; https://redcap.health.uq.edu.au/), hosted by the University of Queensland. Printed paper CRFs will be available if required. All study information and documentation will be securely stored for a period of 15 years after the date of the child's 18 th birthday.

\section{Statistical analysis plan}

\section{Analysis plan}

Analyses will apply the intention-to-treat principle. Descriptive statistics will be used to describe baseline characteristics of the study cohort and each subgroup by treatment group. The primary outcome measure will be analysed using a Mann-Whitney U test as VFD is non-normally distributed variable. Analysis of secondary outcomes includes both comparisons of measurements and proportions, using CIs of differences as the major method of presentation where possible, otherwise standard techniques such as Mann-Whitney $\mathrm{U}$ tests, t-tests and $\chi^{2}$ tests will be utilised. Survival outcomes will be compared between treatment groups using Kaplan-Meier product limit method and log-rank test. Statistical significance will be set at the 0.05 level for the primary outcome.

A safety and efficacy interim analysis after 660 (the half-way point), and after 1000 enrolled patients will be performed by an independent statistician to evaluate for safety endpoints, to assess the predictive probability of reaching the study goals and compare VFD between treatment groups. Consideration to stopping the trial early by an independent DSMB will be based on safety concerns, futility or strong evidence of a difference between groups for VFD (based on a Haybittle-Peto boundary $\mathrm{p}=0.001$ ). A detailed analysis plan specifying statistical analyses including health economic analyses will be placed in the public domain prior to recruitment of the last participant. ${ }^{37}$

\section{Biomarker measurements}

Nested substudies will be performed in selected samples at sites performing biobanking (1) to test the impact of the intervention on markers of systemic and myocardial inflammation, (2) to compare treatment response between patients depending on preintervention severity assessed by markers of inflammation and organ failure and (3) to biochemically define responders to the intervention (to identify patient subgroups prerandomisation that are more likely to respond to a specific treatment). The use of samples/data will be governed by the study steering board and resulting publications must appropriately acknowledge the study. Combining a large RCT with a nested biobank is recommended to maximise scientific knowledge. ${ }^{38}$

\section{Health economic evaluation}

A within trial economic evaluation will be used to determine if providing $\mathrm{NO}$ is cost-saving compared with usual care from the health system perspective. A comprehensive cost-effectiveness analysis will be undertaken to determine the level of cost savings (if any). Length of stay (in PICU and in non-intensive stay) will be the main outcome variable. Resources used before first discharge will be compared between treatments. Resources will subsequently be costed, based on hospital cost centre or standard national sources (eg, Independent Hospital Pricing Authority).

\section{Monitoring}

The study leadership team is responsible for $100 \%$ monitoring of investigator and study nurse credentials, training records and delegation of responsibility logs, and will review $100 \%$ of consent forms. CRFs will be compared with source documentation to ensure data are accurate 
and complete. One hundred per cent of source data verification of eligibility criteria and the primary outcome and the composite secondary outcome of LCOS, ECLS or death will be performed. An independent monitoring per each site will monitor the data fields required for eligibility, primary and secondary study endpoints, and serious adverse events (SAEs) using primary source verification. In addition, site visits and regular monitoring of the blood sample storage will be performed.

\section{Patient and public involvement}

Consumers and the public were consulted to design a video informing parents about the study. Patients and the public had no other involvement in study design.

\section{Ethics}

This protocol and the informed consent document and any subsequent modifications have been reviewed. This study will be conducted in compliance with the current version of the protocol. Any change to the protocol document or informed consent form that affects the scientific intent, study design, patient safety, or may affect a participants' willingness to continue in the study is considered an amendment, and therefore will be written and filed as an amendment to this protocol and/or informed consent form. All protocol deviations must be recorded in the patient record (source document) and on the CRF and must be reported to the principal investigator (PI). Protocol deviations will be assessed for significance by the PI.

Consent will be sought from the parents of every child $<2$ years of age undergoing CPB for elective cardiac surgery over the study period. When the family is seen by the surgeons in preassessment clinic (usually days prior to surgery), the study will be mentioned to them by the surgeon. In addition, the study team will provide study information prior to hospitalisation to families, including printed study flyers, and links to online study documentation (media release). Participant confidentiality is strictly held in trust by the participating investigators, research staff and the sponsoring institution and their agents. The study protocol, documentation, data and all other information generated will be held in strict confidence.

\section{Significance}

Postoperative paediatric cardiac surgical patients have a high consumption of intensive care resources and remain at very high risk of major complications, including cardiac arrest, death and long-term neurological impairment. Approximately $10 \%$ of children with CHD survive with major neurological sequelae postoperatively, resulting in a massive lifelong burden for patients, families, healthcare systems and the society. ${ }^{13}$ An attempt to reduce LCOS and hence perioperative morbidity has the potential to translate not only to a reduction in intensive care resource utilisation but also to impact positively on longterm outcomes. Side effects from heart surgery for CHD translate into long-term morbidity, persisting into adult life with a major impact on other family members and society. This study will deliver the high-level randomised evidence with the potential to show a reduction in postoperative morbidity and mortality in children with CHD.

\section{Author affiliations}

${ }^{1}$ Paediatric Critical Care Research Group, Child Health Research Institute, The University of Queensland, Brisbane, Queensland, Australia

${ }^{2}$ Paediatric Intensive Care Unit, Queensland Children's Hospital, Children's Health Queensland, Brisbane, Queensland, Australia

${ }^{3}$ Cardiac Surgical Unit, Royal Children's Hospital, Melbourne, Victoria, Australia

${ }^{4}$ Faculty of Medicine, Department of Paediatrics, University of Melbourne,

Melbourne, Victoria, Australia

${ }^{5}$ Murdoch Children's Research Institute, Melbourne, Victoria, Australia

${ }^{6}$ Paediatric Intensive Care Unit, Starship Children's Hospital, Auckland, New Zealand

${ }^{7}$ Paediatric Critical Care, Perth Children's Hospital, Western Australia and The University of Western Australia, Crawley, Western Australia, Australia

${ }^{8}$ Kids Critical Care Research, Paediatric Intensive Care Unit, Children's Hospital at Westmead, Westmead, New South Wales, Australia

${ }^{9}$ Sydney Children's Hospital Network, Sydney, New South Wales, Australia

${ }^{10}$ Department of Cardiac Surgery, Royal Children's Hospital, Melbourne, Victoria, Australia

${ }^{11}$ Heart Research, Murdoch Childrens Research Institute, Melbourne, Victoria, Australia

${ }^{12}$ Department of Paediatrics, The University of Melbourne, Melbourne, Victoria, Australia

${ }^{13}$ School of Medicine, Children's Health Clinical Unit, University of Queensland, Brisbane, Queensland, Australia

${ }^{14}$ Cardiac Surgery, Queensland Children's Hospital, Brisbane, Queensland, Australia ${ }^{15}$ Heart Centre for Children, The Children's Hospital at Westmead, Westmead, New South Wales, Australia

${ }^{16}$ Sydney Children's Hospital Network and Faculty of Medicine and Health, University of Sydney, Sydney, New South Wales, Australia

${ }^{17}$ Paediatric Intensive Care Unit, Royal Children's Hospital Melbourne, Melbourne,

Victoria, Australia

${ }^{18}$ Division of Anaesthetics, University Medical Center Utrecht, Utrecht, The Netherlands

${ }^{19}$ Centre for the Business and Economics of Health, The University of Queensland, Brisbane, Queensland, Australia

${ }^{20}$ The Infection and Inflammation Group, The University of Queensland Diamantina Institute, The University of Queensland, Translational Research Institute, Brisbane, Queensland, Australia

${ }^{21}$ The Intensive Care Research Programme, Medical Research Institute of New Zealand, Wellington, New Zealand

${ }^{22}$ School of Public Health, Bond University, Gold Coast, Brisbane, Australia

Acknowledgements The authors would like to thank the parents and children participating in this trial and the medical and nursing and research teams in the participating sites for their help in study setup, recruitment, data collection and monitoring of study data.

Collaborators Warwick Butt, Steve Horton, Johnny Millar, Carmel Delzoppo and Yves d'Udekem: Cardiac and Paediatric Intensive Care Services of Royal Children's Hospital, Melbourne, Australia. John Beca, David Buckley, Taryn Evans, Claire Sherring and John Artrip: Starship Children's Hospital Auckland, New Zealand. Marino Festa, Killian O'Shaughnessy, David Winlaw, Rebecca Fletcher and Simon Byrne: The Children's Hospital at Westmead, Sydney, Australia. Simon Erickson, Sam Barr, Rae Kelly and David Andrews: Perth Children's Hospital, Perth, Australia. Luregn J Schlapbach, Andreas Schibler, Deborah Long, Kerry Johnston, Carla Zuzak, Nelson Alphonso and Benjamin Anderson: Queensland Children's Hospital, Brisbane, Australia. Antje Blumenthal: The University of Queensland Diamantina Institute, Translational Research Institute, The University of Queensland, Brisbane, Australia. Jonas Fooken and Brenda Gannon: Centre for the Business and Economics of Health, The University of Queensland, Brisbane, Australia. Paul Young: Medical Research Institute of New Zealand, Wellington, New Zealand. Mark Jones: School of Public Health, The University of Queensland, Brisbane, Australia. Kim van Loon, Nicole JCW van Belle-van Haaren, Bram van Wijk and Erik Koomen: Wilhelmina Children's Hospital Utrecht, Utrecht, The Netherlands.

Contributors The study protocol first draft was designed by LJS and AS based on the previous pilot study design established by SBH and WB. MJ wrote the section 
on statistical analyses. JF and BG wrote the section on health economic analyses The present study protocol has been revised with input from SBH, DAL, JB, SE, MF, YdU, NA, DW, KJ, CD, KvL, BG, JF, PY, AB, MJ and WB. LJS prepared the final protocol manuscript which was reviewed and approved by all authors.

Funding This work was supported by grants from the Heart Kids Foundation, Australia; the Children's Health Foundation, Brisbane, Australia; and by a grant from the Australian National Health and Medical Research Council (1140322). LJS, YdU and AS hold a NHMRC Clinical Practitioner Fellowship. The Victorian Government's Operational Infrastructure Support Program supported this research project. PY holds a Clinical Practitioner Fellowship from the Health Research Council of New Zealand. Mallinckrodt Pharmaceuticals will provide nitric oxide delivery devices to study centres but has no involvement in study design, conduct, nor analyses.

Competing interests $\mathrm{YdU}$ is a consultant for MSD and Actelion.

Patient consent for publication Not required.

Ethics approval The study was approved by human research ethics committee (Children's Health Queensland, Brisbane, HREC/17/QRCH/43).

Provenance and peer review Not commissioned; externally peer reviewed.

Open access This is an open access article distributed in accordance with the Creative Commons Attribution Non Commercial (CC BY-NC 4.0) license, which permits others to distribute, remix, adapt, build upon this work non-commercially, and license their derivative works on different terms, provided the original work is properly cited, appropriate credit is given, any changes made indicated, and the use is non-commercial. See: http://creativecommons.org/licenses/by-nc/4.0/.

\section{REFERENCES}

1. Triedman JK, Newburger JW. Trends in congenital heart disease: the next decade. Circulation 2016;133:2716-33.

2. Erikssen G, Liestøl K, Seem E, et al. Achievements in congenital heart defect surgery: a prospective, 40-year study of 7038 patients. Circulation 2015;131:337-46. discussion 346.

3. Marelli AJ, Ionescu-Ittu R, Mackie AS, et al. Lifetime prevalence of congenital heart disease in the general population from 2000 to 2010. Circulation 2014;130:749-56.

4. Marelli AJ, Mackie AS, lonescu-Ittu R, et al. Congenital heart disease in the general population: changing prevalence and age distribution. Circulation 2007;115:163-72.

5. Kaltman JR, Andropoulos DB, Checchia PA, et al. Report of the pediatric heart network and national heart, lung, and blood institute working group on the perioperative management of congenital heart disease. Circulation 2010;121:2766-72.

6. Zahler S, Massoudy $\mathrm{P}, \mathrm{Hartl} \mathrm{H}$, et al. Acute cardiac inflammatory responses to postischemic reperfusion during cardiopulmonary bypass. Cardiovasc Res 1999;41:722-30.

7. Levy JH, Tanaka KA. Inflammatory response to cardiopulmonary bypass. Ann Thorac Surg 2003;75:S715-20.

8. Duval EL, Kavelaars A, Veenhuizen L, et al. Pro- and antiinflammatory cytokine patterns during and after cardiac surgery in young children. Eur J Pediatr 1999;158:387-93.

9. Domanski MJ, Mahaffey K, Hasselblad V, et al. Association of myocardial enzyme elevation and survival following coronary artery bypass graft surgery. JAMA 2011;305:585-91.

10. Hoffman TM, Wernovsky G, Atz AM, et al. Efficacy and safety of milrinone in preventing low cardiac output syndrome in infants and children after corrective surgery for congenital heart disease. Circulation 2003;107:996-1002.

11. Duke T, Stocker C, Butt W. Monitoring children after cardiac surgery: a minimalist approach might be maximally effective. Crit Care Resusc 2004;6:306-10.

12. McElhinney DB, Wernovsky G. Outcomes of neonates with congenital heart disease. Curr Opin Pediatr 2001;13:104-10.

13. Ballweg JA, Wernovsky G, Gaynor JW. Neurodevelopmental outcomes following congenital heart surgery. Pediatr Cardiol 2007;28:126-33.

14. Marino BS. New concepts in predicting, evaluating, and managing neurodevelopmental outcomes in children with congenital heart disease. Curr Opin Pediatr 2013;25:574-84.
15. Marino BS, Lipkin PH, Newburger JW, et al. Neurodevelopmental outcomes in children with congenital heart disease: evaluation and management: a scientific statement from the American Heart Association. Circulation 2012;126:1143-72.

16. Brown MD, Wernovsky G, Mussatto KA, et al. Long-term and developmental outcomes of children with complex congenital heart disease. Clin Perinatol 2005;32:1043-57. xi.

17. Bronicki RA, Backer CL, Baden HP, et al. Dexamethasone reduces the inflammatory response to cardiopulmonary bypass in children. Ann Thorac Surg 2000;69:1490-5.

18. Elhoff JJ, Chowdhury SM, Zyblewski SC, et al. Intraoperative steroid use and outcomes following the norwood procedure: an analysis of the pediatric heart network's public database. Pediatr Crit Care Med 2016;17:30-5.

19. Hataishi R, Rodrigues AC, Neilan TG, et al. Inhaled nitric oxide decreases infarction size and improves left ventricular function in a murine model of myocardial ischemia-reperfusion injury. Am J Physiol Heart Circ Physiol 2006;291:H379-84.

20. Chello M, Mastroroberto P, Perticone F, et al. Nitric oxide modulation of neutrophil-endothelium interaction: difference between arterial and venous coronary bypass grafts. J Am Coll Cardiol 1998;31:823-6.

21. Jones SP, Bolli R. The ubiquitous role of nitric oxide in cardioprotection. J Mol Cell Cardiol 2006;40:16-23.

22. Jones SP, Girod WG, Palazzo AJ, et al. Myocardial ischemiareperfusion injury is exacerbated in absence of endothelial cell nitric oxide synthase. Am J Physiol 1999;276:H1567-73.

23. Minamishima S, Kida K, Tokuda K, et al. Inhaled nitric oxide improves outcomes after successful cardiopulmonary resuscitation in mice. Circulation 2011;124:1645-53.

24. Schulz R, Kelm M, Heusch G. Nitric oxide in myocardial ischemia/ reperfusion injury. Cardiovasc Res 2004;61:402-13.

25. Checchia PA, Bronicki RA, Muenzer JT, et al. Nitric oxide delivery during cardiopulmonary bypass reduces postoperative morbidity in children--a randomized trial. J Thorac Cardiovasc Surg 2013;146:530-6.

26. James C, Millar J, Horton S, et al. Nitric oxide administration during paediatric cardiopulmonary bypass: a randomised controlled trial. Intensive Care Med 2016;42:1744-52.

27. Harris PA, Taylor R, Thielke R, et al. Research electronic data capture (REDCap)--a metadata-driven methodology and workflow process for providing translational research informatics support. J Biomed Inform 2009;42:377-81.

28. Gaies MG, Jeffries HE, Niebler RA, et al. Vasoactive-inotropic score is associated with outcome after infant cardiac surgery: an analysis from the Pediatric Cardiac Critical Care Consortium and Virtual PICU System Registries. Pediatr Crit Care Med 2014;15:529-37.

29. Gaies MG, Gurney JG, Yen AH, et al. Vasoactive-inotropic score as a predictor of morbidity and mortality in infants after cardiopulmonary bypass. Pediatr Crit Care Med 2010;11:234-8.

30. Contentin L, Ehrmann S, Giraudeau B. Heterogeneity in the definition of mechanical ventilation duration and ventilator-free days. Am J Respir Crit Care Med 2014;189:998-1002.

31. Cook D, Lauzier F, Rocha MG, et al. Serious adverse events in academic critical care research. CMAJ 2008;178:1181-4.

32. Leteurtre S, Duhamel A, Salleron J, et al. PELOD-2: an update of the PEdiatric logistic organ dysfunction score. Crit Care Med 2013;41:1761-73.

33. Pollack MM, Holubkov R, Funai T, et al. Relationship between the functional status scale and the pediatric overall performance category and pediatric cerebral performance category scales. JAMA Pediatr 2014;168:671-6.

34. Pierrat V, Marchand-Martin L, Arnaud C, et al. Neurodevelopmental outcome at 2 years for preterm children born at 22 to 34 weeks' gestation in France in 2011: EPIPAGE-2 cohort study. BMJ 2017;358:j3448.

35. Noeder MM, Logan BA, Struemph KL, et al. Developmental screening in children with CHD: Ages and Stages Questionnaires. Cardiol Young 2017;27:1447-54.

36. Desai AD, Zhou C, Stanford S, et al. Validity and responsiveness of the pediatric quality of life inventory (PedsQL) 4.0 generic core scales in the pediatric inpatient setting. JAMA Pediatr 2014;168:1114-21.

37. Billot L, Venkatesh B, Myburgh J, et al. Statistical analysis plan for the Adjunctive Corticosteroid Treatment in Critically III Patients with Septic Shock (ADRENAL) trial. Crit Care Resusc 2017;19:183-91.

38. Gustafsson F, Atar D, Pitt B, et al. Maximizing scientific knowledge from randomized clinical trials. Am Heart J 2010;159:937-43. 